\title{
Spectromètre de haute résolution, fort contraste, haute précision
}

\author{
R. Vialla \\ Laboratoire des Verres, UMR 5587, Université Montpellier 2 - C.C. 069, place Eugène \\ Bataillon, 34095 Montpellier cedex 5, France
}

\begin{abstract}
Résumé : Nous présentons la réalisation d'un spectromètre de haute résolution, de fort contraste, haute précision adapté à la mesure de raies Brillouin dans les solides. Il s'agit d'un spectromètre optique constitué de l'association d'un interféromètre de Fabry-Perot plan (FPP) et d'un interféromètre de Fabry-Perot sphérique (FPS). Un signal de référence annexe garantit la précision en fréquence de l'instrument.
\end{abstract}

\section{INTRODUCTION}

Les expériences réalisées au Laboratoire Des Verres portent sur l'étude des pics de diffusion Brillouin dans des milieux transparents. Il s'agit de mesurer l'écart en fréquence par rapport au signal excitateur (laser monomode dans le domaine du visible) et la largeur des pics, caractéristiques directement reliées à la vitesse et à l'atténuation des hypersons dans les matériaux étudiés. L'intensité de ces pics est très faible; les écarts en fréquence sont généralement de quelques $\mathrm{GHz}$ à quelques dizaines de $\mathrm{GHz}$ et la largeur des raies de quelques $\mathrm{MHz}$. De plus on s'intéresse à la variation de ces données en fonction de différents paramètres : nature du matériau, température, position dans l'échantillon. Des variations de vitesse du son de l'ordre de $1 \%$ sont considérées comme de « gros » effets.

Ces particularités entraînent des exigences élevées en terme de luminosité, résolution, contraste, précision des instruments. Nous utilisons des spectromètres interférentiels (FPP tandem 6 passages de J.R. Sandercock) mais les projets d'expériences nouvelles portant sur l'état local de contrainte dans les verres, par exemple, sont hors des limites de ces instruments en terme de résolution et de précision.

\section{PRINCIPE DE L'INSTRUMENT}

Dans sa version initiale, ce spectromètre est constitué de l'association en tandem d'un interféromètre de Fabry-Perot plan fonctionnant en monochromateur (FPP à variation d'indice, fonctionnant en 2 passages) et d'un interféromètre de Fabry-Perot sphérique fonctionnant en analyseur [1]. Il a ensuite évolué vers une version à contraste, résolution et précision améliorés, comportant un signal de référence annexe $[2,3]$. Une réalisation pratique est décrite dans [4].

L'instrument actuel, en cours de validation, a été entièrement reconstruit dans le but de lui apporter stabilité et souplesse d'utilisation. 
La faible intensité du signal à mesurer et la luminosité limitée de l'appareil exigent une durée d'acquisition élevée. Les risques de dérive des dispositifs (dérives thermiques de l'analyseur, du monochromateur, saut de mode du laser), conduisent à effectuer l'acquisition par accumulation de nombreux spectres rapides (typiquement quelques centaines à quelques milliers de spectres d'une seconde chacun). Entre chaque spectre, différents recalages sont réalisés.

La haute résolution $\left(10^{8}\right)$ est obtenue par un FPS d'épaisseur $250 \mathrm{~mm}$. Il comporte 2 miroirs sphériques concaves centrés chacun sur le sommet de l'autre (dispositif dû à P.Connes). L'intervalle spectral libre est alors de $300 \mathrm{MHz}$ et la largeur à mi-hauteur, de 6 $\mathrm{MHz}$ en tenant compte d'une finesse de 50. Mais, par principe, ce dispositif a un contraste limité.

La solution retenue pour atteindre un fort contraste est d'incorporer un monochromateur en amont de l'analyseur : cette fonction est assurée par un interféromètre de Fabry-Perot plan à variation d'épaisseur fonctionnant en multi-passages mais qui ne balaie pas : il est simplement calé sur le maximum du pic Brillouin. Nous faisons passer le faisceau 4 fois sur les lames, ce qui équivaut à 4 FPP en série (mais avec une synchronisation qui ne pourrait être obtenue en pratique avec cette configuration), et permet d'atteindre théoriquement un contraste de $4.10^{1 /}$ avec des lames ayant un pouvoir réflecteur de $91 \%$. Cet interféromètre est animé par des cales piézo-électriques agissant également sur le parallélisme. Un asservissement (d'épaisseur et de parallélisme) est nécessaire afin de recaler entre chaque spectre ce dispositif sur le maximum passant (dérives). Le signal de référence devrait en principe être le signal à mesurer mais sa faible intensité l'interdit. Une des originalités du système est de disposer d'un signal annexe de référence, suffisamment intense et de grande précision en fréquence. Ce signal est obtenu par modulation électro-optique du faisceau laser au moyen d'un générateur hyperfréquence calé sur la fréquence de la raie à mesurer; ce dispositif permet donc une mesure directe des écarts Brillouin et ce à la précision d'une mesure de zéro en s'affranchissant ainsi des erreurs de non-linéarité et d'étalonnage du FPS, soit une précision attendue de $10^{-5}$ avec le générateur actuel $[2,3]$.

Le fonctionnement nécessite un séquencement principalement composé de 3 étapes qui s'enchaînent de manière cyclique : 1) synchronisation de l'analyseur ; 2) asservissement du monochromateur ; 3) mesure.

L'utilisation nécessite évidemment de connaitre au moins approximativement la position de la raie à étudier afin de caler le signal de référence. Cette opération préalable se fait par mesure sur un FPP utilisé seul, en analyseur; pour une garantie de précision, on utilisera un FPP à variation d'indice dont l'intervalle spectral libre est parfaitement connu.

\section{RÉALISATION TECHNIQUE}

Différents dispositifs originaux conçus et réalisés au laboratoire apportent au spectromètre une souplesse d'utilisation : basculement de 2 à 4 passages sur FPP conservant les mêmes positions de faisceau en entrée et sortie, passage aisé du FPP à variation d'épaisseur au FPP à variation d'indice, basculement rapide du FPS 250 à un 100 ou un $50 \mathrm{~mm}$ ou shunt pour analyse sur FPP.

L'acquisition et le séquencement sont effectués par un micro-ordinateur associé à un analyseur multi-canaux déclenché au moyen d'une carte horloge afin de garantir la précision des durées. Le programme, réalisé au laboratoire, en langage C, comporte différentes options de configuration, plusieurs fonctions de test et de simulation afin d'assurer sa maintenance et son développement «hors manip». Hl permet l'enchaînement de séries de spectres avec modification des paramètres de mesure mais sans possibilité d'intervention automatique sur la fréquence du générateur assurant le calage du FPP, actuellement. 


\section{CONCLUSION ET PERSPECTIVES}

Ce spectromètre va permettre de réaliser des expériences très exigeantes en résolution, contraste et précision situées à ce jour hors des limites de notre parc d'instruments.

Nous sommes en train d'équiper l'appareil d'un nouveau générateur hyperfréquence afin d'élargir la gamme de mesure jusqu'à $40 \mathrm{GHz}$. Ce matériel comportera de plus un interface autorisant le pilotage à distance de la fréquence : l'enchaînement de séries de spectres avec recalage automatique du FPP pourra être effectué, répondant ainsi à de nombreux besoins : par exemple la cartographie automatique dans un échantillon.

\section{Références}

[1] Vacher R., thèse Montpellier, 1972

[2] Sussner $H$. and Vacher R., High precision measurements of Brillouin scattering frequencies, Appl. Opt. 18 (1979), 3815-3818.

[3] Vacher R., Sussner H., and Schickfus M. v., A fully stabilized Brillouin spectrometer with high contrast and high resolution, Rev. Sci. Instrum. 51 (3), 1980

[4] Soucat J.M., thèse Montpellier, 1989 